\title{
Predictors of Successful Trial without Catheter for Postoperative Urinary Retention Following Non-Urological Surgery
}

\author{
Kwang Soo Lee, Ki Hong Lim, Sung June Kim, Hyeung Joon Choi, Dong Hoon Noh, Hae Won Lee, Min Chul Cho \\ Department of Urology, Dongguk University Ilsan Hospital, Dongguk University College of Medicine, Goyang, Korea
}

\begin{abstract}
Purpose: To investigate the success rate of trial without catheter (TWOC) for postoperative urinary retention (POUR) after non-urological surgery and to determine predictors of successful TWOC.

Methods: A total of 104 patients who underwent non-urological surgery and were referred to the department of urology for POUR were included in this retrospective study. All eligible patients underwent indwelling catheterization as an initial treatment and then TWOC was performed 3 to 7 days later. POUR was defined as micturition difficulty with greater than $400 \mathrm{~mL}$ of postvoid residual (PVR) urine volume measured by catheterization after non-urological surgery. Successful TWOC was defined as voiding with less than $100 \mathrm{~mL}$ of PVR urine volume. Predictive factors were identified by multivariate regression analysis. All definitions corresponded to recommendations of the International Continence Society.

Results: The mean age of the patients was 65.2 (range, 23 to 92) years. There were 45 male and 59 female patients. Intraoperative indwelling catheterization was performed in $69(66.3 \%)$ patients. Mean duration of indwelling catheterization for POUR was 5.0 (range, 3.0 to 7.0) days and 83 (79.8\%) patients received medication with an alpha-blocker. A successful TWOC was observed in $70(67.4 \%)$ patients. The mean age of the patients with failure of TWOC was significantly higher than that of the patients with successful TWOC. The percentages of female patients, spinal surgery, and prone position during surgery in patients with unsuccessful TWOC were higher than in those with successful TWOC. In the multivariate logistic regression analysis, age and location of surgery (spine vs. non-spine) were the independent predictors of successful TWOC for POUR.

Conclusions: Our data suggest that older age and spinal surgery may be important risk factors for failure of TWOC for POUR after non-urological surgery. Thus, adequate prevention measures may be necessary for POUR after non-urological surgery, especially in patients with these risk factors.
\end{abstract}

Keywords: Urinary retention; Postoperative care; Catheterization

\section{INTRODUCTION}

Postoperative urinary retention (POUR) is a common and potentially serious morbidity but is poorly understood. POUR has generally been defined as the inability to pass any urine in the presence of a palpable or percussible bladder after surgery but the definition varies widely [1]. Its incidence generally ranges from 4 to $25 \%$ [2]. The widely varying reported incidence of POUR reflects differences in patient characteristics, the lack of uniform defining criteria, and the multifactorial etiology of POUR, including age, gender, inadequate perioperative fluids, type of anesthesia and type of surgery [1]. To date, several contributing mechanisms to POUR have been suggested [2]. These include traumatic instrumentation, bladder overdistention, diminished awareness of bladder sensation, decreased bladder contractility, increased outlet resistance, decreased micturition reflex activity, nociceptive inhibitory reflex and preexistent outlet pathology [2]. Several previous studies have reported that factors such as comorbidities, type of surgery, duration of surgery, type of anesthesia or analgesia, amount of intravenous fluid during surgery, and drugs used during the perioperative period, especially anticholinergics or sedatives, can influence the
Corresponding author: Min Chul Cho

Department of Urology, Dongguk University Ilsan Hospital, Dongguk

University College of Medicine, 814 Siksa-dong, Ilsandong-gu, Goyang 410-773, Korea

Tel: +82-31-961-7456 / Fax: +82-31-961-7457 / E-mail: cmc1206@empal.com

Submitted: September 4, 2011 / Accepted after revision: September 23, 2011
This is an Open Access article distributed under the terms of the Creative Commons Attribution Non-Commercial License (http://creativecommons.org/licenses/by-nc/3.0/) which permits unrestricted non-commercial use, distribution, and reproduction in any medium, provided the original work is properly cited. 
development of POUR [1,3-11]. However, most of these studies on the factors influencing POUR showed contradictory results.

Although most patients may recover from POUR with a trial without catheter (TWOC) after a few days of catheterization, some patients with POUR can have persistent urinary retention after failure of TWOC, and this prolongs or complicates the postoperative recovery phase. However, to date, there are sparse data on predictors of recovery from POUR. The aim of the present study was to investigate the success rate of TWOC for POUR after non-urological surgery and to determine the predictors of successful TWOC.

\section{MATERIALS AND METHODS}

\section{Study Design}

This study was approved by the Institutional Review Board at our hospital. Between July 2005 and April 2011, a total of 5,102 patients were referred to the department of urology for urologic problems. Of these, 236 patients complained of micturition difficulty after non-urological surgery. The exclusion criteria were as follows: age younger than 18 years, previous neurological disease, previous urological surgery, and urological disease such as urethral stricture, bladder or prostatic cancer, and benign prostatic hyperplasia (BPH). Thirty-three, 27, and 3 patients were excluded because of previous neurological disease, previous urological surgery, and diagnosis of BPH that may affect micturition after surgery, respectively. After excluding 54 patients with post-void residual (PVR) urine volume of less than $400 \mathrm{~mL}$ measured by catheterization and 15 patients managed by in-and-out catheterization for POUR, a total of 104 patients (45 men and 59 women) with POUR were eligible for this retrospective study. All eligible patients were managed initially in a standard manner with a urethral Foley catheter. TWOC was then performed after 3 to 7 days of indwelling catheterization according to the preference of the individual clinicians. After removal of the urethral Foley catheter, PVR urine volume was evaluated by suitably trained ward nursing staff by using a bladder scanner (BVI-3000 BladderScan, Verathon Inc., Bothell, WA, USA) to determine a need for re-catheterization. This was repeated at convenient intervals until voiding with PVR urine volume of less than $100 \mathrm{~mL}$ was possible or PVR urine volume of greater than $400 \mathrm{~mL}$ was detected by the bladder scanner. When PVR urine volume of greater than $400 \mathrm{~mL}$ was detected by the bladder scanner, re-catheterization was performed. POUR was defined as micturition difficulty with PVR urine volume of greater than $400 \mathrm{~mL}$ measured by catheterization. Successful TWOC was defined as voiding with PVR urine volume of less than $100 \mathrm{~mL}$. All other outcomes were regarded as failures.

A retrospective review was performed to evaluate patient demographics and clinical factors (age, gender, body mass index, comorbidity, presence or absence of lower abdominal discomfort at POUR, preoperative lower urinary tract symptoms or constipation, inability to ambulate at POUR, use of alpha-blocker, duration of indwelling catheterization after POUR, preoperative Modification of Diet in Renal Disease glomerular filtration rate, and presence or absence of preoperative pyuria), surgical factors (duration of surgery, the amount of intravenous fluid administrated during surgery, location of surgery, operative positioning, and presence or absence of intraoperative indwelling catheterization) and anesthetic factors (type of anesthesia, use of patient-controlled anesthesia, and use of anticholinergic drugs during anesthesia). All definitions corresponded to recommendations of the International Continence Society [12].

\section{Data Analysis}

The variables were evaluated for statistical significance by using the Student's t-test and chi square test. To identify the predictors of successful TWOC for POUR, we used logistic regression analysis. Variables with a P-value of less than 0.05 in the univariate analysis were included in the multivariate logistic model. A $5 \%$ level of significance was used for all statistical testing, and all statistical tests were 2-sided. The SPSS ver. 12.0 (SPSS Inc., Chicago, IL, USA) was used for the data analysis.

\section{RESULTS}

The baseline data are shown in Table 1 . The mean age and retention volume of the eligible patients was 65.2 years (range, 23 to 92 years) and $678 \mathrm{~mL}$ (range, 400 to $1,500 \mathrm{~mL}$ ), respectively. There were 45 male and 59 female patients. Intraoperative indwelling catheterization was performed in 69 (66.3\%) patients. Mean duration of indwelling catheterization after POUR was 5.0 days (range, 3.0 to 7.0 days), and 83 (79.8\%) patients received medication with an alpha-blocker. A successful TWOC was observed in $70(67.4 \%)$ patients. Also, a successful TWOC was observed in 36 (74.8\%) of 45 male patients and 34 (57.6\%) of 59 female patients, respectively. Mean age (71.2 \pm 11.6 years) in patients with failure of TWOC was significantly higher than that $(63.1 \pm 16.0$ years) in patients with successful TWOC 
Table 1. Baseline characteristics of the 104 eligible patients

\begin{tabular}{|c|c|c|c|c|}
\hline Variables & Total $(\mathrm{n}=104)$ & $\begin{array}{l}\text { Successful TWOC } \\
\qquad(\mathrm{n}=70)\end{array}$ & $\begin{array}{l}\text { Failure of TWOC } \\
\qquad(\mathrm{n}=34)\end{array}$ & P-value ${ }^{a)}$ \\
\hline \multicolumn{5}{|l|}{ Patient demographics/Clinical variables } \\
\hline Age (yr) & $65.2 \pm 15.3$ & $63.1 \pm 16.0$ & $71.2 \pm 11.6$ & 0.009 \\
\hline$-<70$ & $51(49.0)$ & $38(54.3)$ & $13(38.2)$ & \\
\hline$-\geq 70$ & $53(51.0)$ & $32(45.7)$ & $21(61.8)$ & \\
\hline $\mathrm{BMI}\left(\mathrm{kg} / \mathrm{m}^{2}\right)$ & $24.5 \pm 11.9$ & $24.7 \pm 13.7$ & $23.9 \pm 3.8$ & 0.760 \\
\hline Gender $(\mathrm{M} / \mathrm{F})$ & $45(43.3) / 59(56.7)$ & $36(51.4) / 34(48.6)$ & $9(26.5) / 25(73.5)$ & 0.043 \\
\hline Hypertension & $49(47.1)$ & $33(47.1)$ & $16(47.1)$ & 1.000 \\
\hline Diabetes mellitus & $35(33.7)$ & $22(31.4)$ & $13(38.2)$ & 0.597 \\
\hline Constipation & $12(11.5)$ & $9(12.9)$ & $3(8.8)$ & 0.564 \\
\hline Preop. LUTS & $20(19.2)$ & $12(17.1)$ & $8(23.5)$ & 0.939 \\
\hline Retention volume (mL) & $678 \pm 228$ & $676 \pm 210$ & $683 \pm 279$ & 0.898 \\
\hline Lower abdominal discomfort at POUR & $55(52.9)$ & $38(54.3)$ & $17(50.0)$ & 0.645 \\
\hline Inability to ambulate at POUR & $54(51.9)$ & $36(51.4)$ & $18(52.9)$ & 0.961 \\
\hline Use of alpha-blocker after POUR & $83(79.8)$ & $57(81.4)$ & $26(76.5)$ & 0.560 \\
\hline Duration of indwelling catheterization after POUR & $5.0 \pm 1.1$ & $4.9 \pm 0.9$ & $5.1 \pm 1.4$ & 0.510 \\
\hline Preop. hemoglobin level (g/dL) & $12.0 \pm 2.4$ & $12.1 \pm 2.5$ & $11.8 \pm 1.8$ & 0.641 \\
\hline Preop. platelet count $\left(\times 10^{3} / \mu \mathrm{L}\right)$ & $218.0 \pm 81.8$ & $224.2 \pm 83.7$ & $200.8 \pm 75.3$ & 0.220 \\
\hline Preop. ESR (mm/hr) & $35.8 \pm 25.0$ & $33.0 \pm 23.5$ & $42.1 \pm 33.5$ & 0.421 \\
\hline Preop. CRP (mg/dL) & $7.0 \pm 5.4$ & $6.5 \pm 4.8$ & $7.9 \pm 6.6$ & 0.583 \\
\hline Preop. creatinine (mg/dL) & $0.9 \pm 0.5$ & $0.9 \pm 0.5$ & $0.9 \pm 0.5$ & 0.952 \\
\hline Preop. MDRD GFR (mL/min/1.73m²) & $89.2 \pm 28.1$ & $91.0 \pm 27.0$ & $84.4 \pm 31.1$ & 0.317 \\
\hline No. preop. renal insufficiency $(\%)^{b)}$ & $18(17.3)$ & $10(14.3)$ & $8(23.5)$ & 0.162 \\
\hline Preop. pyuria $(\mathrm{WBC} \geq 5 / \mathrm{HPF})$ & $20(19.2)$ & $14(20.0)$ & $6(17.6)$ & 0.412 \\
\hline \multicolumn{5}{|l|}{ Surgical variables } \\
\hline Duration of surgery (min) & $126.3 \pm 97.8$ & $118.8 \pm 87.7$ & $146.8 \pm 121.2$ & 0.221 \\
\hline Amount of IV fluid administrated during surgery $(\mathrm{mL})$ & $1170.9 \pm 965.9$ & $1083.6 \pm 763.0$ & $1412.0 \pm 1372.4$ & 0.146 \\
\hline Location of surgery & & & & 0.020 \\
\hline Head \& neck & 8 & $6(8.6)$ & $2(5.9)$ & \\
\hline Chest & 1 & $1(1.4)$ & $0(0.0)$ & \\
\hline Upper extremities & 8 & $5(7.1)$ & $3(8.8)$ & \\
\hline Lower extremities & 39 & $29(41.4)$ & $10(2.9)$ & \\
\hline Spine & 15 & $2(2.9)$ & $13(38.2)$ & \\
\hline Abdomen & 32 & $26(37.1)$ & $6(1.8)$ & \\
\hline Pelvic cavity & 1 & $1(1.4)$ & $0(0.0)$ & \\
\hline Operative positioning & & & & 0.010 \\
\hline Supine & $76(73.1)$ & $60(85.7)$ & $16(47.1)$ & \\
\hline Prone & $18(17.3)$ & $5(7.1)$ & $13(38.2)$ & \\
\hline Lateral or others & $10(9.6)$ & $5(7.1)$ & $5(14.7)$ & \\
\hline Intraoperative indwelling catheterization (\%) & $69(66.3)$ & $48(68.5)$ & $21(61.7)$ & 0.233 \\
\hline
\end{tabular}


Table 1. (Continued from the previous page) Baseline characteristics of the 104 eligible patients

\begin{tabular}{lccc}
\hline Variables & Total $(\mathrm{n}=104)$ & $\begin{array}{c}\text { Successful TWOC } \\
(\mathrm{n}=70)\end{array}$ & $\begin{array}{c}\text { Failure of TWOC } \\
(\mathrm{n}=34)\end{array}$ \\
\hline $\begin{array}{l}\text { Anesthetic variables } \\
\text { Type of anesthesia }\end{array}$ & & & \\
$\quad$ P-value ${ }^{\mathrm{a})}$
\end{tabular}

TWOC, trial without catheter; BMI, body mass index; Preop., preoperative; LUTS, lower urinary tract symptoms; POUR, postoperative urinary retention; ESR, erythrocyte sedimentation rate; CRP, C-reactive protein; MDRD GFR, Modification of Diet in Renal Disease glomerular filtration rate (calculated using the abbreviated MDRD study equation); WBC, white blood cell; HPF, high power field; IV, intravenous.

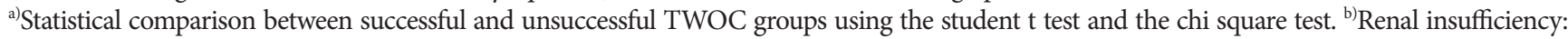
preoperative MDRD GFR less than $60 \mathrm{~mL}$ per minute per $1.73 \mathrm{~m}^{2}$

$(\mathrm{P}=0.009)$. The gender distributions (male/female) were $9 / 25$ and 36/34 in patients with failure of TWOC and successful TWOC, respectively, which was significantly different $(\mathrm{P}=$ 0.043). The distribution of location of surgery (spine/nonspine) in patients with failure of TWOC and successful TWOC was $38.2 \% / 61.8 \%$ and $2.9 \% / 97.1 \%$, respectively, which was significantly different. Also, there was a significant difference in the distribution of operative position (supine/prone/lateral or others) between the two groups (47.1\%/38.2\%/14.7\% vs. $85.7 \% /$ 7.1\%/7.1\%). Urodynamic study was performed in 11 of 34 patients with failure of TWOC. Detrusor overactivity, detrusor underactivity, acontractile detrusor, and normal detrusor function were observed in $3,4,5$, and 2 patients, respectively. During indwelling catheterization, $3(1.9 \%)$ and $10(9.6 \%)$ patients complained of gross hematuria and urethral discomfort, respectively. However, no patient had serious catheter-related complications such as febrile urinary tract infection.

In the univariate logistic regression analysis, age, gender (male vs. female), location of surgery (spine vs. non-spine), and operative position (supine vs. prone) were significantly associated with successful TWOC (Table 2). In the multivariate logistic regression analysis, age and location of surgery (spine vs. non-spine) were the independent predictors of successful TWOC for POUR (odds ratio [OR], 0.962; $\mathrm{P}=0.048$, and OR, $0.140 ; \mathrm{P}=0.038$ ) (Table 2). Mean age and gender distribution (male/female) in the 15 patients who underwent spinal surgeries were $65.5 \pm 12.2$ years and 5/10, respectively. Also, all patients underwent spinal surgeries in the prone position.

According to retention volume, the patients were stratified into 2 subgroups: those with a retention volume of $\geq 600 \mathrm{~mL}$ and those with a retention volume of $<600 \mathrm{~mL}$. The value selected $(600 \mathrm{~mL})$ to divide the patients into the two subgroups is the maximum capacity of the bladder in an adult [13]. In the subgroup of 58 patients with a retention volume of $600 \mathrm{~mL}$ or greater, successful TWOC was observed in 45 (77.6\%) patients. The percentage $(69.2 \%)$ of female patients among 13 patients with failure of TWOC was significantly higher than that (42.2\%) among 45 patients with successful TWOC $(\mathrm{P}=0.048)$. The proportion $(7.7 \%)$ of patients who underwent intraoperative indwelling catheterization in the failed TWOC group was significantly lower than that (57.8\%) in the successful TWOC group $(\mathrm{P}=0.023)$. Also, mean age $(70.8 \pm 7.1$ years $)$ in patients with failed TWOC was significantly higher than that $(63.2 \pm 13.5$ years $)$ in those with successful TWOC $(\mathrm{P}=0.048)$. In the multivariate logistic regression analysis, age and presence or absence of intraoperative indwelling catheterization were the independent predictors of successful TWOC for POUR (OR, 0.968, $\mathrm{P}=0.049$; OR, 8.475, $\mathrm{P}=0.045$ ) (Table 3). In the subset of patients with retention volume of $\geq 600 \mathrm{~mL}$, those who did not receive indwelling catheterization during surgery were at an 8.5 times higher risk of failed TWOC than were those with intraoperative indwelling catheterization (Table 3).

In the subset of 46 patients with retention volume of less than $600 \mathrm{~mL}$, successful TWOC was observed in 32 (69.6\%) patients. In the univariate logistic regression analysis, age, gender (female vs. male), location of surgery (spine vs. non-spine), and operative position (prone vs. supine) significantly influenced successful TWOC (OR, 0.923, P=0.036; OR, 0.575, 
Table 2. Univariate and multivariate predictors of successful TWOC for POUR after non-urological surgery in the total patients

\begin{tabular}{|c|c|c|c|c|}
\hline & OR $(95 \% \mathrm{CI})$ & P-value & Adjusted OR (95\% CI) & P-value \\
\hline \multicolumn{5}{|l|}{ Patient demographics/clinical variables } \\
\hline Age (yr) & $0.960(0.926-0.995)$ & 0.027 & $0.962(0.927-0.997)$ & 0.048 \\
\hline $\mathrm{BMI}\left(\mathrm{kg} / \mathrm{m}^{2}\right)$ & $1.008(0.960-1.058)$ & 0.762 & & \\
\hline Gender $(\mathrm{M} / \mathrm{F})$ & & 0.048 & & 0.144 \\
\hline Male & 1.000 & & 1.000 & \\
\hline Female & $0.367(0.136-0.989)$ & & $0.456(0.159-1.309)$ & \\
\hline Hypertension & $0.900(0.358-2.263)$ & 0.823 & & \\
\hline Diabetes mellitus & $0.682(0.250-1.857)$ & 0.454 & & \\
\hline Constipation & $1.010(0.970-1.050)$ & 0.950 & & \\
\hline Preop. LUTS & $0.946(0.230-3.888)$ & 0.939 & & \\
\hline Retention volume (mL) & $0.996(0.990-1.002)$ & 0.897 & & \\
\hline Lower abdominal discomfort at POUR & $1.286(0.515-3.211)$ & 0.589 & & \\
\hline Inability to ambulate at POUR & $0.977(0.392-2.438)$ & 0.961 & & \\
\hline Use of alpha-blocker after POUR & $1.433(0.426-4.808)$ & 0.562 & & \\
\hline Duration of indwelling catheterization after POUR & $0.930(0.751-1.152)$ & 0.506 & & \\
\hline Preop. renal insufficiency ${ }^{a}$ & $2.542(0.700-9.231)$ & 0.156 & & \\
\hline Preop. Pyuria & $0.604(0.180-2.028)$ & 0.414 & & \\
\hline \multicolumn{5}{|l|}{ Surgical variables } \\
\hline Duration of surgery (min) & $0.997(0.993-1.002)$ & 0.229 & & \\
\hline Amount of IV fluid administrated during surgery $(\mathrm{mL})$ & $1.000(0.999-1.001)$ & 0.500 & & \\
\hline Location of surgery & & 0.028 & & 0.038 \\
\hline Non-spinal & 1.000 & & 1.000 & \\
\hline Spinal & $0.154(0.206-0.903)$ & & $0.140(0.022-0.901)$ & \\
\hline Operative positioning & & 0.010 & & 0.113 \\
\hline Supine & 1.000 & & 1.000 & \\
\hline Prone & $0.111(0.302-0.390)$ & & $0.142(0.020-1.006)$ & \\
\hline Lateral or others & $0.250(0.058-1.070)$ & & $0.263(0.057-1.219)$ & \\
\hline Intraoperative indwelling catheterization & $1.362(0.831-3.460)$ & 0.137 & & \\
\hline \multicolumn{5}{|l|}{ Anesthetic variables } \\
\hline Type of anesthesia & & 0.836 & & \\
\hline Spinal or epidural & 1.000 & & & \\
\hline General & $0.902(0.340-2.392)$ & & & \\
\hline Use of patient-controlled anesthesia & $0.569(0.258-2.107)$ & 0.569 & & \\
\hline Duration of patient-controlled anesthesia (day) & $0.573(0.695-1.223)$ & 0.573 & & \\
\hline Use of anticholinergic drugs during anesthesia & $0.964(0.379-2.456)$ & 0.939 & & \\
\hline
\end{tabular}

TWOC, trial without catheter; POUR, postoperative urinary retention; OR, odds ratio; CI, confidence interval; BMI, body mass index; Preop., preoperative; LUTS, lower urinary tract symptoms; IV, intravenous.

${ }^{a}$ Renal insufficiency: preoperative Modification of Diet in Renal Disease glomerular filtration rate less than $60 \mathrm{~mL}$ per minute per $1.73 \mathrm{~m}^{2}$. 
Table 3. Multivariate predictors of successful TWOC for POUR in 58 patients with retention volume of $\geq 600 \mathrm{~mL}$

\begin{tabular}{lcc}
\hline & Adjusted OR (95\% CI) & P-value \\
\hline Age $(\mathrm{yr})$ & $0.968(0.938-0.998)$ & 0.049 \\
$\begin{array}{l}\text { Gender }(\mathrm{M} / \mathrm{F}) \\
\text { Male }\end{array}$ & 0.274 \\
$\quad$ Female & 1.000 & \\
$\begin{array}{l}\text { Intraoperative indwelling cath- } \\
\text { eterization }\end{array}$ & $0.474(0.113-1.709)$ & \\
\hline
\end{tabular}

TWOC, trial without catheter; POUR, postoperative urinary retention.

$\mathrm{P}=0.047 ; \mathrm{OR}, 0.755, \mathrm{P}=0.048 ; \mathrm{OR}, 0.145, \mathrm{P}=0.043)$, but the presence or absence of intraoperative indwelling catheterization was not significantly associated with successful TWOC (OR, $1.847, \mathrm{P}=0.409)$. However, in the multivariate analysis, age was the only independent predictor of successful TWOC for POUR (OR, 0.959, $\mathrm{P}=0.047$ ) (Table 4).

Twenty of 34 patients with failure of TWOC were discharged from the hospital with indwelling catheters and the remaining 14 patients performed clean intermittent catheterization (CIC) after being discharged. The decision regarding whether the patients had indwelling catheterization or CIC was determined by individual doctor-patient consultation. A second TWOC was performed 1 to 2 weeks after discharge from the hospital. Eight of 20 patients with indwelling catheters at discharge had success in the second TWOC.

\section{DISCUSSION}

Although it is well known clinically that POUR is a frequent and potentially serious condition that can prolong hospital stay, limited data is available regarding the predictors of outcome of TWOC for POUR. The present study extends the current state of knowledge in the study of POUR by further exploring the outcome of TWOC and factors influencing success in patients with POUR after non-urological surgery. The important findings of our study can be briefly summarized as follows: 1) older age and spinal surgery can be risk factors for unsuccessful TWOC for POUR, and 2) the presence or absence of intraoperative indwelling catheterization may be an important factor influencing the success of TWOC in patients with a retention volume of $\geq 600 \mathrm{~mL}$ but not in those with a retention volume of $<600 \mathrm{~mL}$.

The contractility of the detrusor decreases with advancing age [2]. Accordingly, in light of previous studies, it was generally
Table 4. Multivariate predictors of successful TWOC for POUR in 46 patients with retention volume of $<600 \mathrm{~mL}$

\begin{tabular}{lcc}
\hline & Adjusted OR (95\% CI) & P-value \\
\hline Age $(\mathrm{yr})$ & $0.959(0.923-0.995)$ & 0.047 \\
Gender (M/F) & 0.235 \\
Male & 1.000 & \\
Female & $0.952(0.892-1.012)$ & 0.168 \\
Location of surgery & 1.000 & \\
Non-spinal & $0.847(0.686-1.008)$ & \\
Spinal & 1.000 & 0.153 \\
Operative positioning & & \\
Supine & $0.244(0.124-1.110)$ & \\
Prone & $0.161(0.016-1.632)$ & \\
Lateral or others &
\end{tabular}

TWOC, trial without catheter; POUR, postoperative urinary retention; $\mathrm{OR}$, odds ratio; $\mathrm{CI}$, confidence interval.

assumed that POUR increases with age, with the risk increasing by 2.4 to 2.8 times in patients over 50 years of age [3,5-7]. Furthermore, previous studies have demonstrated that younger age is significantly associated with recovery from POUR $[14,15]$. In accordance with these previous studies, our results showed that age was an independent predicting factor of successful TWOC for POUR and that patients older than 70 years were at a 1.8 times higher risk of failure of TWOC than were those younger than 70 years (data not shown).

Controversy has existed about the impact of gender on the development of POUR. Some studies have shown that gender is not correlated significantly with the development of POUR $[5,10,11]$. Also, Tammela et al. [14] suggested that there was no significant difference in frequencies of persistent micturition difficulty after the first catheterization for POUR between male and female patients. Other studies reported a higher incidence of POUR in men than in women [3,9]. In contrast, a previous study demonstrated that female gender was an independent risk factor for POUR after surgery for benign anorectal disease in a multivariate analysis [8]. Similarly, our data showed that female gender was significantly associated with failure of TWOC in the univariate regression model. There are a few explanations for this. First, voiding could have been hindered in the women because they were lying supine on the bed, which led to a more uncomfortable voiding position in the women than in the men [8]. Second, women might feel more reluctant to void in an unfamiliar surrounding without privacy compared with men [8]. Third, because women are liable to cystitis anatomically, this 
could have an adverse impact on recovery of micturition from POUR [15]. Unfortunately, gender was not an independent predictor of successful TWOC for POUR in the multivariate model of the present study. Thus, larger cohort studies are warranted to further elucidate the impact of gender on outcomes of TWOC for POUR.

The duration of catheterization before TWOC for urinary retention is still an area of debate. A previous study by Djavan et al. [16] reported that a successful TWOC was achieved in $44 \%$ of patients with in-and-out catheterization, in $51 \%$ of those with 2 days of indwelling catheterization and in $62 \%$ of those with 7 days of indwelling catheterization, which suggests that prolonged catheterization may usually be associated with a greater success rate of TWOC. In contrast, other studies have not demonstrated an impact of duration of catheterization on TWOC outcome $[17,18]$. Taube and Gajraj [17] found that in patients randomly allocated to three groups (urethral catheters were removed either immediately after the bladder was emptied, or 24 or 48 hours later), there was no significant difference in the rates of successful TWOC among the three groups. Recently, Emberton and Fitzpatrick [18] suggested that the benefits associated with a duration of catheterization of more than 3 days appear to be marginal in terms of TWOC success (59.6\%) compared with a shorter duration of catheterization (56.4\%). In line with these studies, the present study did not demonstrate a prognostic significance of duration of catheterization in terms of TWOC outcome for POUR. Furthermore, because all patients in our study underwent catheterization of $\geq 3$ days and the percentage of patients with catheterization of 3 days was only $11.5 \%$, we could not confirm whether duration of catheterization was associated with the outcome of TWOC for POUR.

Previous studies have shown that a drained volume at catheterization of $\geq 1 \mathrm{~L}$ could be a risk factor for unsuccessful TWOC, which suggests a correlation between retention volume at POUR and outcome of TWOC $[14,16,18]$. However, our data did not demonstrate an influence of retention volume on outcome of TWOC for POUR. In our series the relatively small number of cases $(9.5 \%)$ with a drained volume at catheterization of more than $1 \mathrm{~L}$ might be a reason that no statistical significance as an independent prognostic marker was attained.

The incidence of POUR can vary according to the location of surgery [1]. The present study showed that the patients with spinal surgery were at a higher risk of unsuccessful TWOC for POUR than were those with non-spinal surgery. This may be because they usually need prolonged bed rest and cannot urinate in the correct voiding position after spinal surgery [7]. In this study, the patients who underwent surgeries in the prone position had a higher probability of unsuccessful TWOC than did those who underwent surgeries in the supine position, but this variable lost statistical significance in the multivariate analysis.

Interestingly, although in a subset of patients with retention volume of $\geq 600 \mathrm{~mL}$, the presence of intraoperative indwelling catheterization was an independent predictor of successful TWOC for POUR, there was no significant correlation between the two in patients with a retention volume of $<600 \mathrm{~mL}$. Even a single episode of bladder over-distention may lead to the deposition of collagen between the muscle fibers of the detrusor, decreasing the contractility and leading to impairment of bladder emptying [19]. Furthermore, bladder ischemia may contribute to the persistent impairment of contractility after bladder overdistension [20]. Thus, avoidance of excessive bladder distention by indwelling catheterization during surgery might be important for recovery of micturition from POUR in patients with a retention volume over $600 \mathrm{~mL}$. This can be further supported by the experimental observation of a reduced bladder response to sacral neural stimulation during over-distention and after over-distention [21].

Some caution may need to be applied when interpreting our data. First, the present study is limited by its retrospective nature and therefore carries with it all the inherent potential issues associated with such studies. Systematic evaluation with urodynamic analysis was performed only in a portion of the patients with POUR. Also, although the medical history of preoperative lower urinary tract symptoms was taken by an urologist at the urology consultation, some asymptomatic patients with chronic urinary retention might have been included in this study, thus confounding our analysis. Second, the number of eligible patients may be insufficient to detect all the factors significant for predicting the outcome of TWOC in patients with POUR.

Our study is appropriate relevant to clinical decision making. The results of this study give insight into a common but poorly explored postoperative problem, which is POUR following non-urological surgery. These results can be helpful in establishing a treatment protocol and prevention measures for patients with POUR after non-urological surgery.

In conclusion, our data suggest that older age and spinal surgery may be important risk factors for failure of TWOC for POUR after non-urological surgery. Thus, clinicians should 
pursue preventive measure such as systemic monitoring of PVR urine volume by bladder scanner to avoid POUR and minimize the associated morbidity, especially in patients with these risk factors. Also, research into an adequate treatment protocol for POUR should be further encouraged. The present study indicates that indwelling catheterization during surgery may be needed to avoid excessive bladder distention and persistent micturition difficulty. These data may be substantially important to clinicians caring for such patients. However, prospective studies with a larger cohort are necessary to confirm these findings.

\section{CONFLICT OF INTEREST}

No potential conflict of interest relevant to this article was reported.

\section{REFERENCES}

1. Baldini G, Bagry H, Aprikian A, Carli F. Postoperative urinary retention: anesthetic and perioperative considerations. Anesthesiology 2009;110:1139-57.

2. Wein AJ. Lower urinary tract dysfunction in neurologic injury and disease. In: Wein AJ, Kavoussi LR, Novick AC, Partin AW, Peters CA, editors. Campbell's-Walsh urology. 9th ed. Philadelphia: Saunders; 2007. p. 2040-306.

3. Tammela T, Kontturi M, Lukkarinen O. Postoperative urinary retention. I. Incidence and predisposing factors. Scand J Urol Nephrol 1986;20:197-201.

4. Darrah DM, Griebling TL, Silverstein JH. Postoperative urinary retention. Anesthesiol Clin 2009;27:465-84.

5. Keita H, Diouf E, Tubach F, Brouwer T, Dahmani S, Mantz J, et al. Predictive factors of early postoperative urinary retention in the postanesthesia care unit. Anesth Analg 2005;101:592-6.

6. Lau H, Lam B. Management of postoperative urinary retention: a randomized trial of in-out versus overnight catheterization. ANZ J Surg 2004;74:658-61.

7. Lee SJ, Kim YT, Lee TY, Woo YN. Analysis of risk factors for acute urinary retention after non-urogenital surgery. Korean J Urol 2007; 48:1277-84.

8. Toyonaga T, Matsushima M, Sogawa N, Jiang SF, Matsumura N,
Shimojima Y, et al. Postoperative urinary retention after surgery for benign anorectal disease: potential risk factors and strategy for prevention. Int J Colorectal Dis 2006;21:676-82.

9. Zaheer S, Reilly WT, Pemberton JH, Ilstrup D. Urinary retention after operations for benign anorectal diseases. Dis Colon Rectum 1998;41:696-704.

10. Kumar P, Mannan K, Chowdhury AM, Kong KC, Pati J. Urinary retention and the role of indwelling catheterization following total knee arthroplasty. Int Braz J Urol 2006;32:31-4.

11. Petros JG, Bradley TM. Factors influencing postoperative urinary retention in patients undergoing surgery for benign anorectal disease. Am J Surg 1990;159:374-6.

12. Abrams P, Cardozo L, Fall M, Griffiths D, Rosier P, Ulmsten U, et al. The standardisation of terminology of lower urinary tract function: report from the Standardisation Sub-committee of the International Continence Society. Neurourol Urodyn 2002;21:167-78.

13. Abrams P. Urodynamic techniques. In: Abrams P, editor. Urodynamics. London: Springer-Verlag; 2005. p. 117-46.

14. Tammela T, Kontturi M, Lukkarinen O. Postoperative urinary retention. II. Micturition problems after the first catheterization. Scand J Urol Nephrol 1986;20:257-60.

15. Yoon YE, Lee JW, Park SY, Park HY, Lee TY, Kim YT. Predictive factors for recovery from acute urinary retention after non-urogenital surgery. Korean J Urol 2009;50:976-81.

16. Djavan B, Shariat S, Omar M, Roehrborn CG, Marberger M. Does prolonged catheter drainage improve the chance of recovering voluntary voiding after acute retention of urine (AUR)? Eur Urol 1998; 33(Suppl 1):110.

17. Taube M, Gajraj $H$. Trial without catheter following acute retention of urine. Br J Urol 1989;63:180-2.

18. Emberton M, Fitzpatrick JM. The Reten-World survey of the management of acute urinary retention: preliminary results. BJU Int 2008;101 Suppl 3:27-32.

19. Hinman F. Editorial: postoperative overdistention of the bladder. Surg Gynecol Obstet 1976;142:901-2.

20. Kitada S, Wein AJ, Kato K, Levin RM. Effect of acute complete obstruction on the rabbit urinary bladder. J Urol 1989;141:166-9.

21. Bross S, Schumacher S, Scheepe JR, Zendler S, Braun PM, Alken P, et al. Effects of acute urinary bladder overdistension on bladder response during sacral neurostimulation. Eur Urol 1999;36:354-9. 